\title{
Practical Application of Two-Way Multiple Overlapping Relationships in a BDM Network
}

\author{
Seon-Gyoo Kim \\ Department of Architectural Engineering, Kangwon National University, Gangwon-do 200-701, South Korea
}

\begin{abstract}
Today, most construction projects in urban environments are complex high-rise buildings that present unique challenges, including local building ordinances and restrictions, adjoining public and residential areas, narrow sidewalks and streets, and underground utilities, all of which require extensive planning and tight schedules. A major problem facing such projects is to formulate realistic schedules that will make it possible to meet contractual completion dates with limited resources and budgets. The scheduling software products currently used in construction projects, which include Primavera P6, Microsoft Project, etc., are not actually applied as a scheduling tool in practical construction projects, which instead generally depend on Microsoft Excel or a bar-chart. This is because the existing scheduling programs cannot provide more user-oriented schedule format such as representing two-way multiple overlapping relationships. To overcome this deficiency, the BDM (beeline diagramming method) is proposed as a new networking technique in 2010. But two-way multiple overlapping relationships generate the loop in a conventional schedule computation process. This paper addresses the loop phenomenon of two-way multiple overlapping relationships in a BDM network as well as proposes the solutions of them, and then presents a practical application of two-way multiple overlapping relationships at a real project.
\end{abstract}

Key words: Critical path method, beeline diagramming method, schedule computation, two-way multiple overlapping relationships, consecutive activities.

\section{Introduction}

Big construction projects in today's crowded urban environments are not straightforward, especially in major cities such as New York, Mexico City, Seoul, London and Tokyo. Most construction projects in these large metropolitan areas tend to consist of high-rise buildings designed to house or provide offices and workplaces for large concentrations of people. These high-rise building projects are very complicated and involve unique challenges and opportunities. Construction teams must deal with local building ordinances and restrictions, adjoining public and residential areas, narrow sidewalks and streets, and underground utilities, all of which necessitate a high level of planning and scheduling [1]. In general, a key feature of any construction schedule is to create

Corresponding author: Seon-Gyoo Kim, professor, research field: construction management (schedule/risk). E-mail:sg1208@kangwon.ac.kr. a realistic sequence of construction activities that are laid out in a time-related logical order to enable workers to complete the project on time and on budget. This schedule is the basic tool that guides and coordinates the progress of a project by measuring, monitoring, and controlling the construction progress. Schedules are increasingly important for every player in construction due to their ability to integrate the individual tasks that make up the overall construction process efficiently and effectively to create a seamless whole.

Various domestic and international scheduling software products have been used in construction projects in South Korea, including Primavera P6, Microsoft Project, Neo Plan and Open Plan. However, these software tools are not actually applied to the practical construction management scheduling process, and most construction projects instead depend on Microsoft Excel or manual bar-chart methods with CAD (computer-aided design) software. However, 
although a schedule formulated in Excel will allow project managers to understand the overall process involved in their construction project, this approach is not compatible with the CPM (critical path method), which shows the linkages between predecessor and successor activities as a whole network to support better scheduling. Microsoft Excel is simply not an effective scheduling tool [2].

This need for better schedules and control methods has led to the development of new network scheduling approaches that facilitate and support the complex processes involved in major urban construction projects. There are currently three networking techniques in use: the PERT (program evaluation and review technique), the CPM - these two techniques are also known as ADM (arrow diagram methods), and the PDM (precedence diagram method). Most of the commercially available scheduling software packages are based on either ADM or PDM. Kim [3] indicated that the efficacy of CPM network scheduling techniques has been proven for a number of construction projects and it is now recognized as a useful tool, but many project participants who are not construction managers, scheduling experts or original users continue to have difficulty in understanding and utilizing CPM techniques. Kim [4] pointed out that CPM process scheduling does not accurately reflect every activity at the construction site, and it is often difficult to visually and clearly recognize the construction progress status. Additionally, CPM techniques are not able to integrate activity and budget progresses effectively. In general, CPM/PERT scheduling techniques were developed with an underlying concept of minimizing total project duration [5]. Therefore, most large construction projects in South Korea either do not utilize CPM network scheduling or use it only unwillingly at the request of the project owner and many scheduling activities still depend on the bar-chart method. As these results demonstrate, as yet the available scheduling methods fail to provide a dynamic tool capable of measuring, monitoring, and controlling the progress of a project. Additionally, multipoint projects can be scheduled using commonly accepted CPM techniques, but continuous utilization of resources across units cannot be assured when these CPM networks are used [6].

It has been suggested that a new CPM network technique, the BDM (beeline diagramming method), may offer a way to overcome the difficulties and deficiencies associated with existing CPM techniques. The most distinctive technique that sets BDM apart from existing methods is its ability to make a linkage between any points during simultaneous predecessor and successor activities. This linkage greatly simplifies the display of two overlapping activities, and the linkage can extend and establish a multiple relationship between any pair of predecessor and successor activities when an activity has a long duration or several completion times during the activity. The multiple linkages in BDM are not limited to displaying the start and completion times of activities, but can also connect any point during those activities as well as allowing multiple overlapping relationships between activities.

To apply either single or two-way multiple overlapping relationships for two consecutive activities to the existing methods, a loop in the network is created to prevent the schedule updating. In general, a loop is a circular relationship between two activities in a network, where each is both a predecessor and a successor to the other. A loop constitutes an error that must be corrected for CPM calculations [7, 8]. For instance, when a revised schedule is generated to reflect forthcoming activities, the network repeatedly computes both an ESD (early start date) and an EFD (early finish date) but is not able to update the completion day. The objective of the paper is to address the loop phenomenon of two-way multiple overlapping relationships in a BDM network as well as propose the solutions of them, and then present a practical application of two-way multiple 
overlapping relationships at a real project.

\section{Research Scope and Methods}

The objective of this research is to propose a practical application of two-way multiple overlapping relationships in a BDM network. This paper consists of the following tasks that are required to compute a construction project schedule:

First is to identify and describe the general concept and calculation principles of a BDM network, which is a CPM scheduling technique. Second is to analyze and suggest possible solutions to the loop problem in order to permit the computation of a completion day and thus facilitate appropriate schedule updates. Third is to verify two-way multiple overlapping relationships in a BDM network and present a practical application of two-way multiple overlapping relationships at a real project.

The analysis and validation of the proposed solution to the loop problem presented in this paper will provide a firm foundation for the development of new and more effective tools that facilitate scheduling activities on construction sites.

\section{Beeline Diagramming Method}

\subsection{Basic Concept, Principle, and Characteristics}

In 2010, the BDM was proposed as a new network scheduling technique [9]. The basic concept of the $\mathrm{BDM}$ is to represent the overlapping relationship of two consecutive activities by the shortest straight line, with an arrow to represent the direction of work flow. The BDM connects any point of the predecessor activity to any point of the successor activity in a network scheduling. The linkage between the two activities defines the "beeline" between them, named after the path taken by bees returning to their hive which is supposedly the shortest and most direct route, to simplify the overlapping relationships. The BDM has only one principle: it represents the single or multiple overlapping relationships of two consecutive activities in the network by one or more beelines in any circumstance.

\subsection{Linkage Representation Types in the BDM}

Unlike in the PDM, linkage relationships in the BDM can be represented at any middle point between two consecutive activities. The PDM, in contrast, represents linkage relationships only by FS, SS, FF, and SF relationships, with lead-time between the starting and finishing points. This research proposes three types of linkage representations in the BDM. The first is the "N-N" type shown in Fig. 1a, which represents two consecutive activities that are mutually connected at any point (in days) after their respective starts.

The first "N" in Fig. 1a refers to the days that have elapsed from the start date of the preceding activity; the second " $\mathrm{N}$ " refers to the days that have elapsed from the start date of the succeeding activity; and the "-" linking them is the separation indicator between the two Ns. An example of the first representation type is illustrated in Fig. 1b. In this figure, two consecutive activities in the BDM are connected by a "7-4" type, which indicates a link between a point 7 days after the start date of the preceding Activity A and a point 4 days after the start date of the succeeding Activity B.

The second type is " $<\mathrm{N}>$ ", shown in Fig. 2a, where the successor starts some days after the completion of the predecessor.

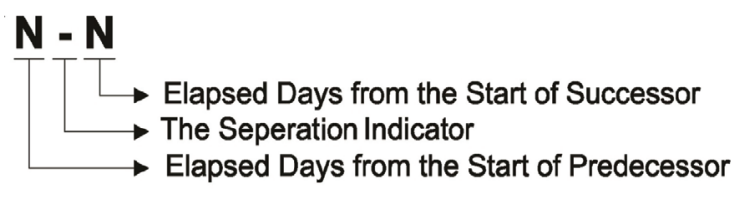

(a)



(b)

Fig. 1 “N-N": (a) representation type; (b) example. 
The "N" in Fig. 2a refers to the lead-time between the completion of the preceding activity and the start of the succeeding activity. The opening " $<$ " and closing " $>$ " indicate the lead-time space indicators. An example of the FS representation type is illustrated in Fig. 2b. In the figure, two consecutive activities in the BDM are connected by " $<4>$ ", which indicates that the succeeding Activity B starts 4 days after the preceding Activity A has been completed.

The third type represents two-way multiple overlapping relationships between two consecutive activities in terms of the elapsed days as shown in Fig. 3. This type will provide more flexible and accurate relationships on the intermediate milestones of two consecutive activities that may have a long duration. However, their two-way linkages may cause inevitable loop phenomenon in the schedule computation process.

\section{Schedule Computation for Two-Way} Multiple Overlapping Relationship

\subsection{The Loop Phenomenon in Two-Way Multiple Overlapping}

As mentioned earlier, one of the advantages of BDM techniques is their ability to deal with two-way multiple overlapping relationships in a scheduling network. However, the linkage in two-way multiple overlapping relationships frequently generates a loop that causes the schedule computation process to endlessly circulate around the same route. This means that BDM is not able to calculate and schedule a date for subsequent activities because its ESD, EFD, LSD, and LFD are repetitively calculated through the BDM linkages. The multiple overlapping relationships of two consecutive activities can be classified into two cases: Case 1, where one successor follows one predecessor, as shown in Fig. 4, and Case 2, where two successors follow one predecessor, as shown in Fig. 5.

Fig. 4 represents Case 1 for two-way multiple overlapping relationships. The loop problem affecting the schedule computation process occurs whenever two activities, $\mathrm{A}$ and $\mathrm{B}$, have multiple linkages (a), (b), (c), (d), as shown in Figs. 4 and 5.

On the forward-pass computation, the ESD of Activity A has been decided by its predecessors, after which the next forward computation is sequentially performed from linkage (a) to (b) based on the predefined ESD. The sequential forward computation from (a) to (b) triggers the loop because Activities

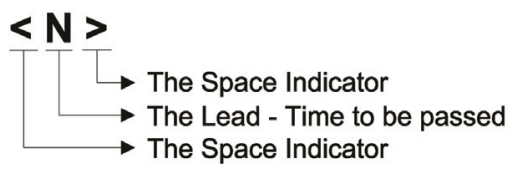

(a)

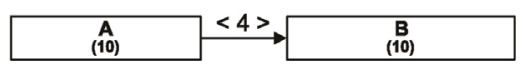

(b)

Fig. 2 "<N>": (a) representation type; (b) example.

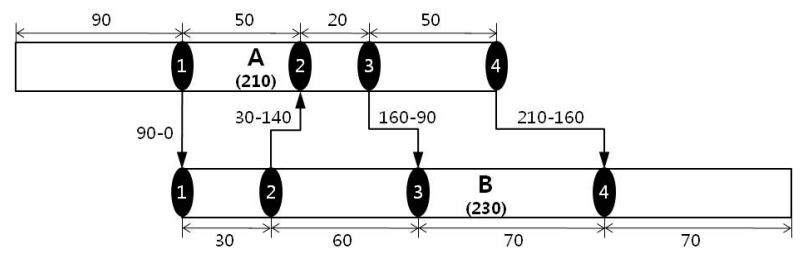

Fig. 3 Two-way multiple overlapping relationships.

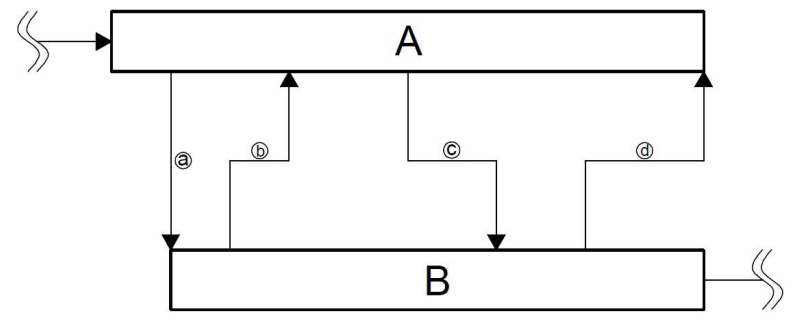

Fig. 4 Two-way multiple overlapping relationships (Case 1).

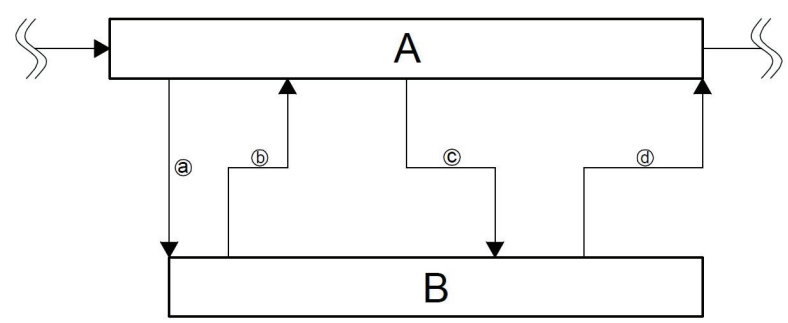

Fig. 5 Two-way multiple overlapping relationships (Case 2). 
$\mathrm{A}$ and $\mathrm{B}$ have two-way multiple overlapping relationships. This means that the ESD for Activity B will be calculated based on the original ESD for activity A due to the linkage (a) between Activity A and Activity B, but then the ESD of Activity A will be recalculated due to the linkage (b), after which the ESD of activity $B$ will be recalculated based on the new value for the ESD of Activity A and so on. As a result of these repeated calculations, the forward pass computation is unable to move on to the next activities.

This loop phenomenon also occurs on the backward pass computation. The LSD for Activity B in Case 1, shown in Fig. 4, is first decided by the successor activities, after which the LSD of Activity A is calculated by linkage (a) based on the predefined LSD of Activity B and then the LSD of Activity B is recalculated by linkage (b) and so on.

For Case 2, shown in Fig. 5, the LSD of Activity A is originally decided by its successor activities. The LSD of Activity B is then calculated by linkage (b) based on the predefined LSD of Activity A, which leads to the LSD of Activity A being recalculated and by linkage (a), once again triggering the loop phenomenon.

\subsection{Proposed Solutions to the Loop Problem}

\subsubsection{Forward Pass Computation}

The diagram shown in Fig. 6 is an example of the forward pass computation for Case 1 and shows two-way multiple BDM linkages between Activities A and B, labeled here as "2-0", "2-6", "10-11", and "14-20". The ESD of Activity A is assumed to be "10". In order to prevent a loop occurring for the two-way multiple overlapping relationships during the forward computation, the ESD of a successor should be calculated using a minimum lag value among the linkages between Activities $\mathrm{A}$ and $\mathrm{B}$ from the predecessors toward a successor. For instance, if the
ESD of Activity B is calculated by a linkage "2-0" with a lag value " 0 " and a linkage " $10-11$ " with a lag value " 3 " among the linkages from Activity A to Activity B, it is calculated as " $10+2-0=12$ " and " $10+10-11=9$ " for the two linkages, respectively, by the BDM forward calculation Equation (2) ${ }^{1}$ [4]. As a result, any linkages with more than the smallest lag value can be ignored in the computation of the ESD.

Furthermore, to prevent the loop phenomenon occurring in the forward pass computation, the linkages, which retrogress from a successor to a predecessor and thus trigger the loops, need to be ignored. This is done by specifying that ESDs may not be greater than the ESD first calculated by a predecessor, as the continual recalculating of ESDs performed by the linkages generates the loops. In the example shown in Fig. 6, the linkages that generate the loops are "2-6" and "14-20" and are reversed from Activity B to Activity A. If the ESDs of Activity A are recalculated by Equation (2) based on these two linkages, they become " $12+2-6=8$ " and " $12+14-$ $20=6$ ", respectively. This means that the ESDs of Activity A that has been recalculated by the linkages, thus potentially generating the loops, can equal the first calculated ESD of the activity but not exceed it. Therefore, the ESD of a predecessor would be selected as the maximum value among the ESDs calculated during the forward pass computation. Any linkages that generate loops will then be ignored in calculating the ESD in the network schedule, and the

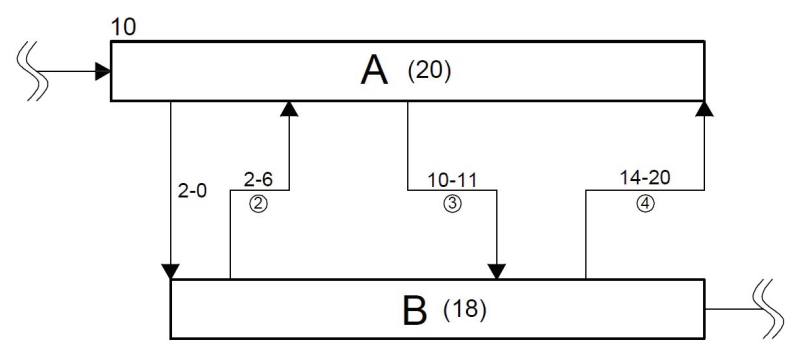

Fig. 6 Forward computation for Case 1.

${ }^{1}$ Equation (2): $E F D_{J}=E S D_{J}+D_{J}$, where, $E F D_{J}$ : early finish date of Activity $\mathrm{J}, E S D_{J}$ : early start date of Activity $\mathrm{J}, D_{J}$ : duration of Activity J. 
linkages will simply represent the mutual relationships between two activities.

\subsubsection{Backward Pass Computation}

The diagram in Fig. 7 is an example of the backward pass computation for Case 1 and shows the two-way multiple BDM linkages between Activities A and B as "2-1", "3-7", "10-11", and "15-18”.

The forward pass computation has already been performed for the network diagram. In general, the backward pass computation for the network obtains LSD by simply reversing the direction of the arrows. The LSD of a successor must be calculated using the linkage with the minimum lag value among the linkages going from A to B. In Fig. 7, the LSDs of Activity A are calculated to be " $13+1-2=12$ " and " $13+11-10=14$ " from the BDM backward calculation Equation (4) ${ }^{2}$ [3] based on the two linkages "2-1" and "10-11", which have lag values of " 0 " and " 2 ", respectively. Comparing the results of this calculation, any LSD computation that exceeds the minimum lag value yields a higher value for LSD and can therefore be ignored for the network schedule computation.

In order to avoid triggering the loop phenomenon in the backward pass computation, it is again necessary to disregard all linkages that retrogress from a successor to a predecessor, as in the forward pass computation. This means that it is impossible to have smaller LSDs than the LSD originally defined even though LSDs continue to be recalculated by the linkages, thus generating loops. In Fig. 7, the linkages that generate loops are "3-7" and "15-18". If the LSDs of Activity B are recalculated by Equation (4) based on these two linkages, they yield " $12+7-3=16$ " and " $12+18-25=5$ ", respectively. Thus, the LSDs of Activity $B$ that continue to be recalculated by the linkages, and thus generate loops, could be equal to the LSD originally calculated for the activity, but cannot become smaller than this value. As before, the

${ }^{2}$ Equation (4): $L F D_{I}=L S D_{I}+D_{I}$, where, $L F D_{J}$ : late finish date of Activity $\mathrm{J}, L S D_{J}$ : late start date of activity $\mathrm{J}, D_{J}$ : duration of Activity $\mathrm{J}$
LSD of a successor is taken to be the minimum value obtained for all the LSDs calculated for the backward computation. Accordingly, any linkages that generate loops can be ignored when calculating the LSD for the network schedule, and the linkages simply represent the mutual relationships between the two activities.

The backward computation procedure for Case 2, shown in Fig. 8, requires a different computation method because here the Successor B does not connect to the next successors but instead connects to its Predecessor A only. To apply a forward pass computation, ESD and LSD must therefore be calculated for linkage "2-1", which has the minimum lag value among the linkages between Activities A and B. However, the LSD for Successor B cannot be calculated for the linkages from Predecessor A to Successor B because the LSD of Successor A has already been calculated, which occurs before the LSD of Successor B is found from the backward computation. It is therefore not possible to ignore the linkages from Successor B to its Predecessor A. In this case, the LSD and LFD of Successor B must be calculated for the linkage with the minimum lag value among the linkages from Successor B to Predecessor



Fig. 7 Backward computation for Case 1.

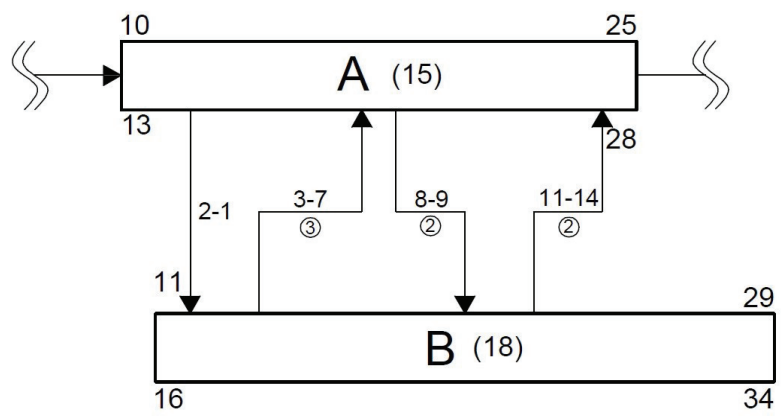

Fig. 8 Backward computation for Case 2. 


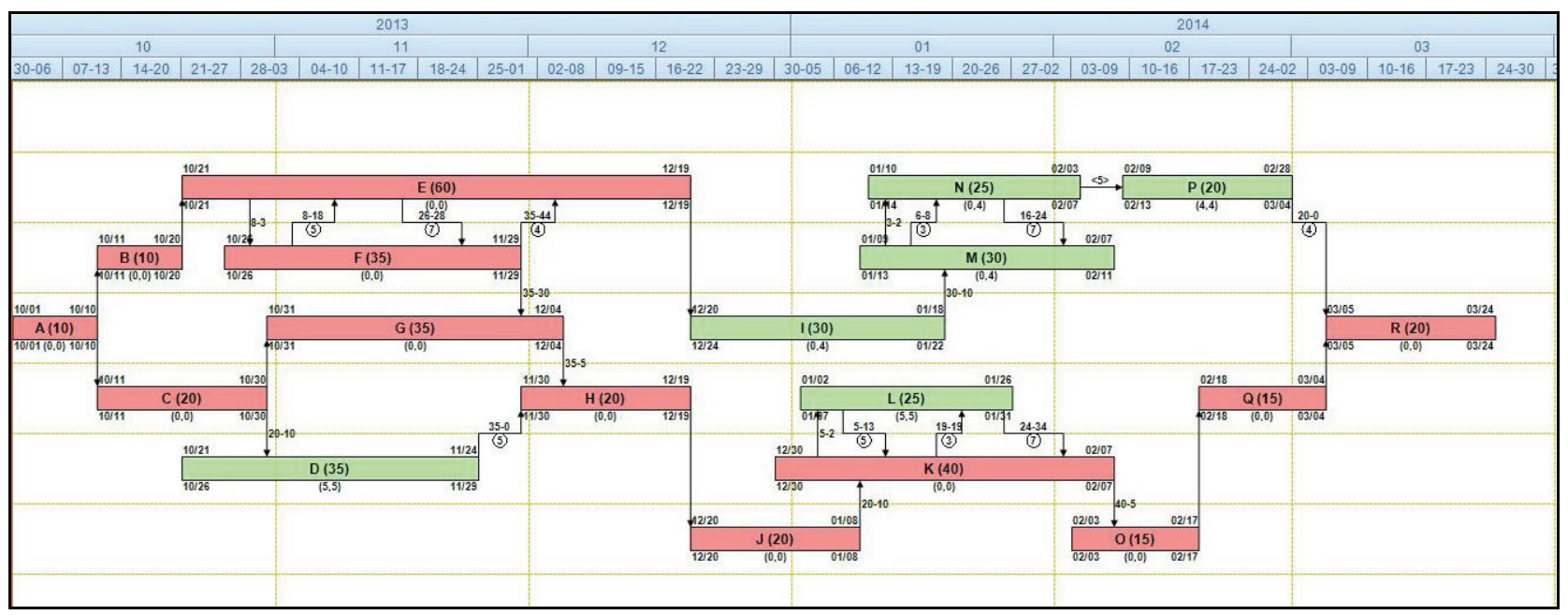

Fig. 9 BDM sample network for validation.

A. For instance, if the LSD of Successor B is calculated by linkage " $3-7$ " to have a lag value " 3 " and by linkage "11-14" to have a lag value " 2 ", the LSDs for the two linkages are then calculated as " $13+$ $7-3=17$ " and " $13+14-11=16$ ", respectively, by Equation (4). Therefore, the LSD and LFD of Successor B must be calculated for the linkage with a minimum lag value among all the linkages that retrogress from a successor to a predecessor in cases where a successor has two-way multiple overlapping relationships with a single predecessor only.

\section{Validation of Schedule Computation for Two-Way Multiple Overlapping Relationships Using BDM Sample Network}

To validate new schedule computation processes for two-way multiple overlapping relationships, a sample BDM network composed of 18 activities was created using Beeliner Pro ${ }^{3}$, as shown in Fig. 9. The sample network includes all three types of two-way multiple overlapping relationships, including linkages between Activities $\mathrm{E}$ and $\mathrm{F}$ where both a predecessor and a successor are connected to another successor, linkages between Activities $\mathrm{K}$ and $\mathrm{F}$ where a predecessor is only connected to another successor, and linkages between Activities $\mathrm{M}$ and $\mathrm{N}$ where a

\footnotetext{
${ }^{3}$ Scheduling software operated by the BDM technique.
}

successor is only connected to another successor. The BDM network schedule computation for this sample network was accomplished successfully, with no conflicts arising. This exercise clearly demonstrates the validity of the new schedule computation method proposed for solving the troublesome loop phenomenon plaguing two-way multiple overlapping relationships in BDM networks.

In order to examine the schedule computation procedure for the sample network shown in Fig. 9 in more detail, the cases of the two-way multiple overlapping relationships for E-F and M-N, where a successor is connected to another successor are considered. The schedule computation for E-F and $\mathrm{M}-\mathrm{N}$ is performed by ignoring any linkages except the linkage that has the minimum lag value among all the linkages from the predecessor to the successor.

In Fig. 9, Predecessor E and Successor F are joined by the linkages " $8-3$ " and " $8-18$ ", and Predecessor F is also connected to another successor. In this case, the forward pass computation to obtain the ESD of Successor F requires the ESDs of Successor $F$ to be calculated as "Oct. $21+8$ days -3 days $=$ Oct. 26" and "Oct. $21+26$ days -28 days = Oct. 19" by Equation (1) based on linkage "8-3" with a lag value " 0 " and linkage "26-28" with a lag value "7", respectively. Once it has been verified that the linkage with the smallest lag value produces the latest ESD 
after the forward pass computation, those linkages that have larger lag values must be ignored. When conducting the backward pass computation to obtain the LSD of Predecessor E, the LSDs of Predecessor E are calculated as "Oct. $26+3$ days -8 days $=$ Oct. 21 " and "Oct. $26+28$ days -26 days $=$ Oct. 28 " by Equation (4) based on linkage "8-3" with a lag value " $0 "$ and linkage "26-28" with a lag value " 7 ", respectively. Once again, it must be verified that the backward pass computation has been performed by the linkage with the smallest lag value. Recalculating the LSDs of Activity F for the linkages " $8-18$ " and "35-44", both of which would trigger loops, yields "Oct. $26+18$ days -8 days $=$ Nov. 5 " and "Oct. $26+$ 44 days -35 days $=$ Nov. 4", which are both later than the LSD originally calculated, "Oct. 26". This confirms that the linkages that trigger loops should be ignored.

The second loop phenomenon causing problems in the sample network shown in Fig. 9 is for Predecessor $\mathrm{K}$ and Successor L, which have four linkages, "5-2", "5-13", "19-19", and "24-34". Successor L is connected only to Predecessor K. In this case, the ESDs of Successor L are calculated as "Dec. $30+5$ days -2 days $=$ Jan. 2 " and "Dec. $30+19$ days -19 days = Dec. 30" by Equation (1) based on linkage "5-2", with a lag value " 0 ", and linkage "19-19", with a lag value "3", respectively. According to the network schedule computation, a linkage with a big lag value can be ignored for the forward pass computation, even where a successor is not connected to another successor. The backward pass computation gives the LSD of Predecessor K, but the LSD of Predecessor $\mathrm{K}$ has already been calculated to be earlier than that of Successor L. In this case, the successor is not connected to another successor so the LSD of Successor L would be obtained using the linkages from Successor L to Predecessor $\mathrm{K}$ that trigger loops. The linkages responsible for the loops are linkage "5-13", with a lag value " 5 ", and linkage "24-34", with a lag value "7". Calculating the LSDs of Successor L using Equation (4) for these two linkages yields "Dec. $30+13$ days -5 days $=$ Jan. 7" and "Dec. $30+34$ days -24 days = Jan. 9". This confirms that for cases where a successor is not connected to another successor, the backward computation must be performed by the linkage that has the smallest lag value among the linkages related to the loops.

\section{Practical Application of Two-Way Multiple Overlapping Relationships at the Real Project}

Top or high-level managers who have had extensive experiences at the real projects can usually grasp the project status based on the summarized project information promptly. But there is a lot of possibility for misunderstanding or misjudgment if they cannot get exact or enough information from the lower levels. Therefore, project management team must always try to provide more accurate summarized information to them.

By the same token, schedulers at the construction project always make his best for providing more exact and reliable schedule information in the summarized format to the top managers. If the summarized schedule shows logics or critical paths between the summarized activities as same as the CPM schedule does, top managers can understand a project status more accurately and conveniently. However, current scheduling techniques such as ADM and PDM cannot provide CPM logics at summarized schedule levels, on the other hand, BDM technique can provide the CPM logics on the summarized schedules applying by two-way multiple overlapping relationships as described at this paper.

Fig. 10 shows a BDM network for the finish works of apartment unit that consists of 21 activities with various BDM linkages at WBS (work breakdown structure) Level 4

Fig. 11 is a BDM schedule of Level 3 summarized from levEl 4 of Fig. 10 that shows many two-way 


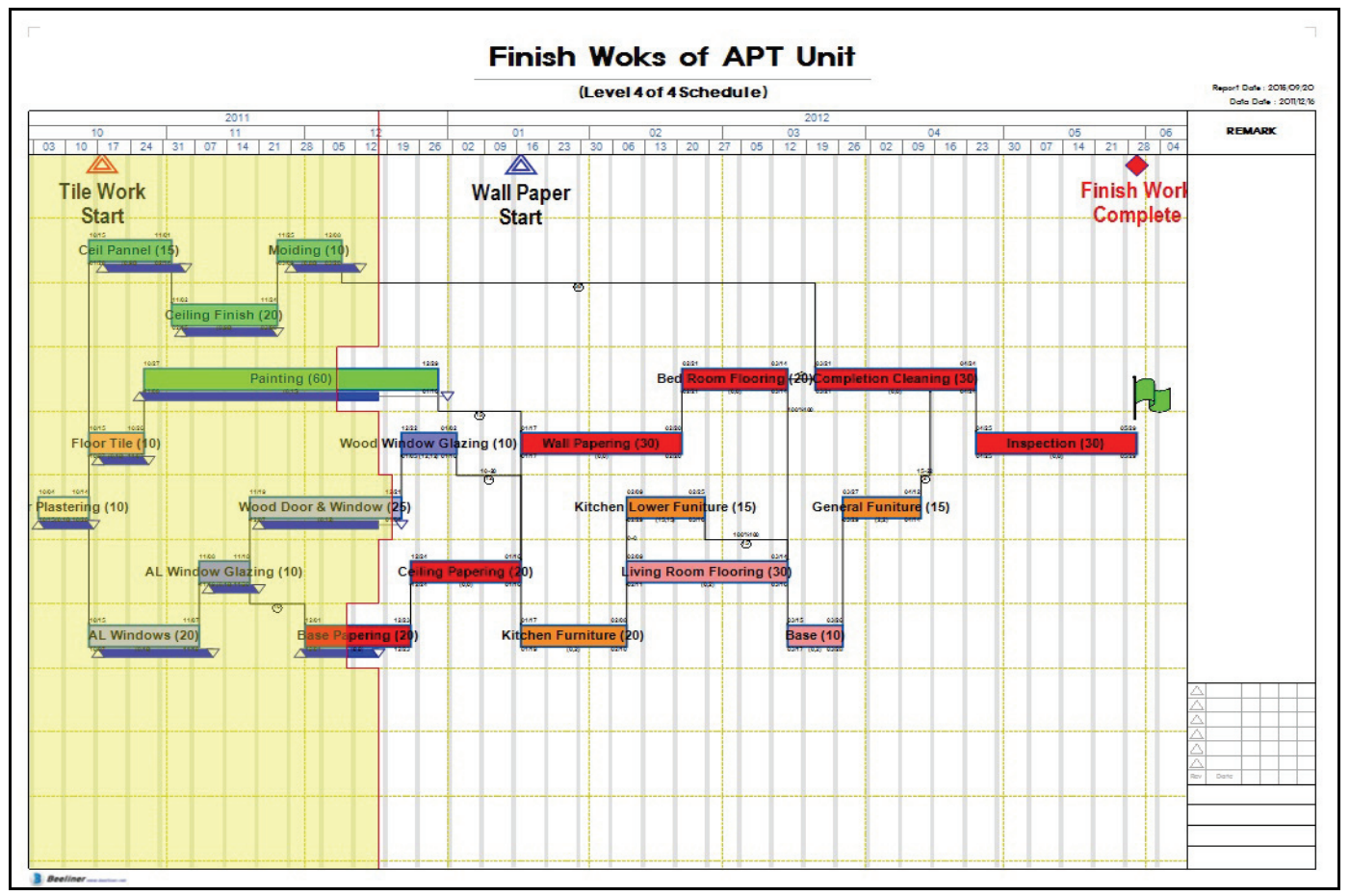

Fig. 10 BDM network at WBS Level 4.

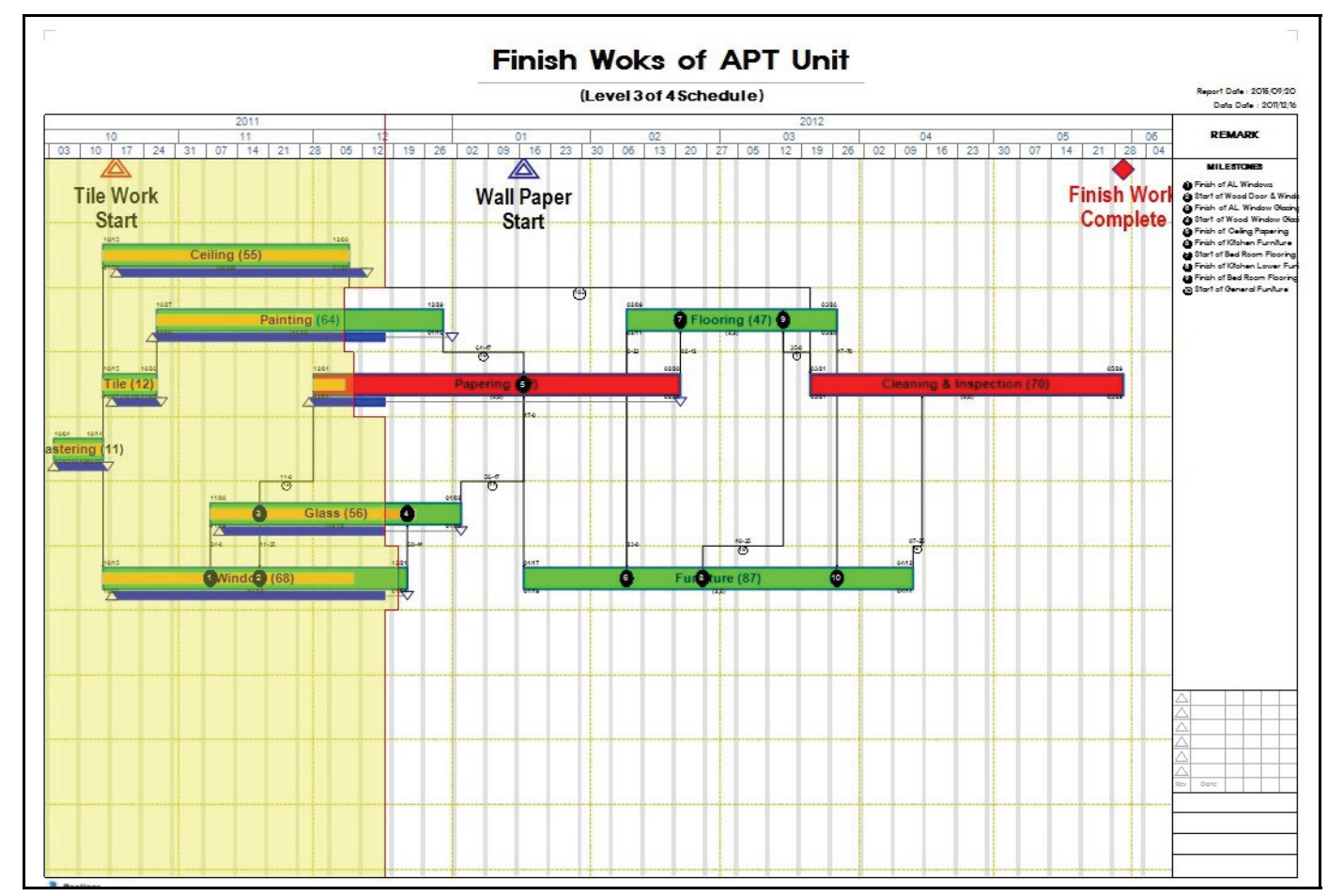

Fig. 11 Summarized BDM network at WBS Level 3. 


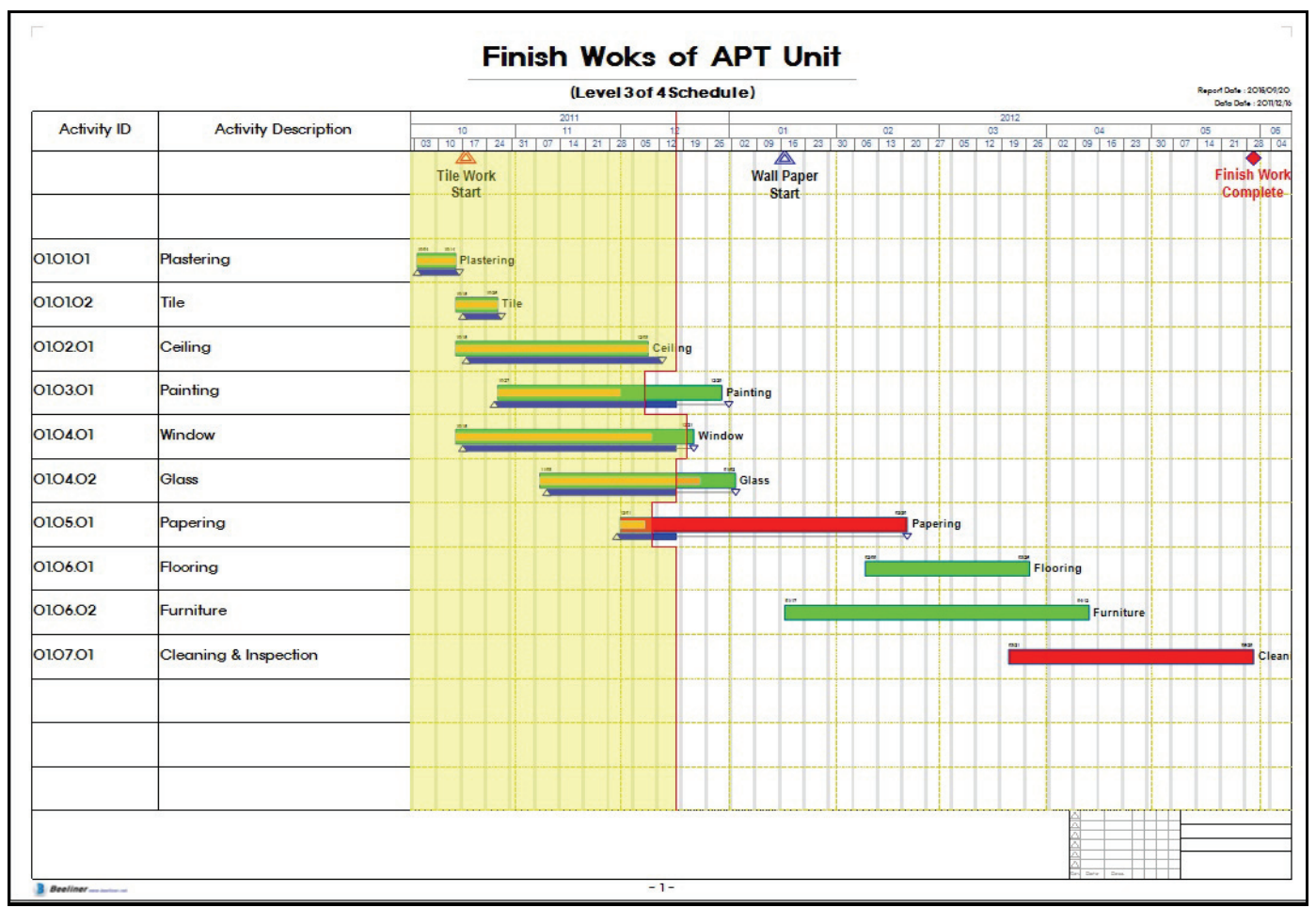

Fig. 12 Typical bar-chart at WBS Level 3.

multiple overlapping relationships between the summarized 10 activities. Remark area at the top-left side of Fig. 11 shows many intermediate milestones with the black circled numbers and descriptions in order for top managers to understand two-way multiple overlapping relationships on the summarized schedule more exactly and conveniently.

Fig. 12 is a typical bar-chart schedule of Level 3 without CPM logics between activities generated by Beeliner Pro. Although a bar-chart has a lot of advantages due to its simplicity and understandability, a CPM schedule becomes more and more acceptable because a bar-chart cannot provide critical path or sub-critical path as well as analysis schedule impacts and anticipate schedule delays. Accordingly, it is so natural that top managers will accept a summarized BDM schedule with CPM logics such as Fig. 11 more actively than a bar-chart without any logic such as Fig. 12.

\section{Discussion and Conclusions}

The beeline diagramming method was introduced in
2010 as a new scheduling technique that utilizes links to simplify the display of two overlapping activities and establishes a multiple relationship between any pair of predecessor and successor activities to overcome the problems while retaining the benefits of existing ADM and PDM techniques. Above all, the BDM technique represents the multiple relationships effectively and efficiently when an activity has a long duration and includes several completion times. In general, the linkages for these two-way multiple overlapping relationships may trigger a loop that causes the schedule computation process to endlessly circulate around the same route between two consecutive activities and prevent the scheduling of a completion date. To address this issue, this study investigated the causes of the loop phenomenon for the case of two-way multiple relationships in a BDM network, proposed a new scheduling computation method and demonstrated its application with two case studies, and validated the proposed method with a BDM network.

Construction schedules can be a useful tool for 
managing, controlling, and supporting the complex processes involved in any construction projects. The network schedule can define all activity interrelationships and durations while simultaneously taking into account the resources available. It can also reflect the actual work done and be used to minimize the disruption involved in incorporating approved changes into the as-planned schedule. However, it is questionable whether projects that are currently underway are capable of fully utilizing this type of network scheduling as a dynamic tool capable of measuring, monitoring, and controlling the progress of a project. The two-way multiple overlapping relationships of the BDM network schedule proposed in this study provides an important window into the general process functions of logical links in network schedules. The suggested BDM technique has the potential to make a significant contribution to the development of new network scheduling approaches that facilitate and support the complex processes involved in today's urban construction projects.

\section{References}

[1] Lambeck, R., and Eschemuller, J. 2009. Urban Construction Project Management. New York, NY: McGraw Hill.

[2] Son, J.-J. 2003. "A Field Study on the Operational Realities of Process Control Software." Journal of the Architectural Institute of Korea 19 (12): 23-31.

[3] Kim, K.-H. 2004. "CPM Barchart Technique for Construction Scheduling." Korean Journal of Construction Engineering and Management 5 (5): 58-64.

[4] Kim, S.-G. 2011. "CPM Schedule Summarizing Function of the Beeline Diagramming Method." Journal of Asian Architecture and Building Engineering 11 (2): 101-9.

[5] Elazouni, A. M., and Gab-Aliah, A. A. 2004. "Finance-Based Scheduling of Construction Projects Using Integer Programming." Journal of Construction Engineering and Management 130 (1): 15-24.

[6] Harris, R., and Ioannou, P. G. 1998. "Scheduling Projects with Repeating Activities." Journal of Construction Engineering and Management 124 (4): 269-78.

[7] Callahan, M. T. 1992. Construction Project Scheduling. New York, NY: McGraw-Hill.

[8] Mubarak, S. 2010. Construction Project Scheduling and Control. 2nd ed. Hoboken, NJ: John Wiley \& Sons, Inc.

[9] Kim, S.-G. 2010. Advanced Networking Technique. Seoul, South Korea: Kimoondang. 